PO078

\title{
DETECTOR POSITIONING SYSTEM WITH SIX DEGREES OF FREEDOM FOR THE EXTENSION OF A PHOTOMETRIC BENCH
}

\author{
Johannes Ledig et al.
}

DOI 10.25039/x46.2019.PO078

from

CIE x046:2019

Proceedings

of the

29th CIE SESSION

Washington D.C., USA, June 14 - 22, 2019

(DOI 10.25039/x46.2019)

The paper has been presented at the 29th CIE Session, Washington D.C., USA, June 14-22, 2019. It has not been peer-reviewed by CIE.

\section{(C) CIE 2019}

All rights reserved. Unless otherwise specified, no part of this publication may be reproduced or utilized in any form or by any means, electronic or mechanical, including photocopying and microfilm, without permission in writing from CIE Central Bureau at the address below. Any mention of organizations or products does not imply endorsement by the CIE.

This paper is made available open access for individual use. However, in all other cases all rights are reserved unless explicit permission is sought from and given by the CIE.

CIE Central Bureau

Babenbergerstrasse 9

A-1010 Vienna

Austria

Tel.: +43 17143187

e-mail: ciecb@cie.co.at

www.cie.co.at 


\title{
DETECTOR POSITIONING SYSTEM WITH SIX DEGREES OF FREEDOM FOR THE EXTENSION OF A PHOTOMETRIC BENCH
}

\author{
Ledig, J., Schrader, C., Lindner, D., Sperling, A. \\ Physikalisch-Technische Bundesanstalt (PTB), Bundesallee 100, 38116 Braunschweig, GERMANY \\ johannes.ledig@ptb.de
}

DOI 10.25039/x46.2019.PO078

\begin{abstract}
The traceable calibration of an Imaging Luminance Measurement Device (ILMD) requires a detailed characterization as well as an estimation of uncertainty contributions. Both are related to the spatial and angular characteristic, therefore a variable but precisely known positioning of the ILMD in front of a light source is needed. This is realized by extending a photometric bench facility by an articulated industrial robot dedicated for absolute positioning of an ILMD. Emphasis is given to make also the robot cell itself capable for photometric measurements. The working space of the installed system, aspects regarding differences to the common use of such industrial robots, and issues of an ILDM as a tool are discussed.
\end{abstract}

Keywords: imaging luminance measuring device, luminance distribution, near-field goniophotometry

\section{Motivation, specific objective}

The traceable calibration of an Imaging Luminance Measurement Device (ILMD) or of the luminance distribution of a light source requires knowledge about numerous contributions to measurement errors and uncertainties. Typically, a light source with a uniform luminance is used to characterize the relative non-uniformity of the photo-response (PRNU) (Bünger et al., 2015). In case of a non-uniform luminance, a sequence of translation can be used (Ferrero et al., 2014). For keeping a fixed distance, a translation parallel to the light source, cf. Figure 1 , is suitable. But the determined PRNU will still include artefacts from a non-ideal directionality of the source, e.g. if it is not a perfect Lambertian emitter (Schrader and Sperling, 2016).
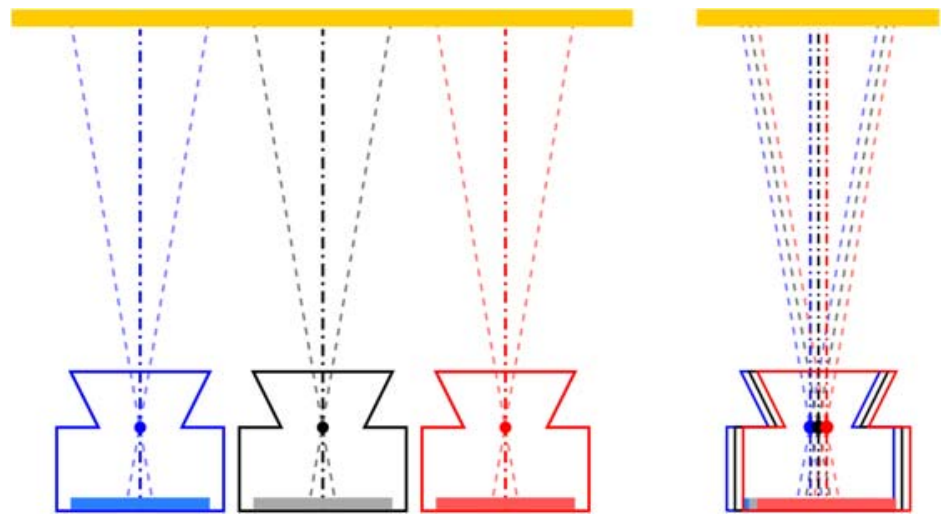

Figure 1 - scheme of the ILMD pose with projection paths (dashed) of exemplary pixels, coloured corresponding to a sequence of large (left) and small (overlapping, right) translation.

A PRNU determined from a sequence of rotation around the ILMD projection centre, cf. Figure 2 , does not include such artefacts of the luminance directionality. But it requires a more versatile positioning system and knowledge about the projection of each pixel, e.g. position and direction. In combination with a geometric referencing by photogrammetry, e.g. the light source position and a determination of the ILMD projection centre and imaging distortions, inhomogeneities of the ILMD (PRNU) and light source (luminance distribution) can be separated from another. This was already demonstrated using a positioning system with five degrees of freedom (Schrader 
and Sperling, 2016). By rotating the ILMD around the light source, cf. Figure 2, the (local) luminance directionality can be determined.
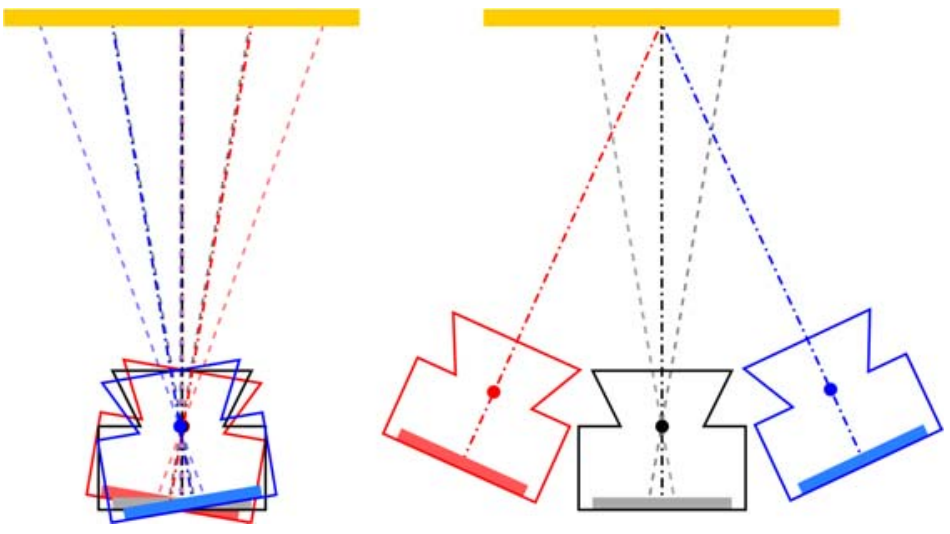

Figure 2 - scheme of the ILMD pose with projection paths (dashed) of exemplary pixels, coloured corresponding to a rotation around the ILMD projection centre (left) and around the light source (right).

In order to characterize ILMDs, especially those with a large measurement field angle (e.g. up to $90^{\circ}$ ), therefore a large range of its pose (position and direction) with respect to a light source is required. Such a pose sequence can only be achieved by an automated positioning system with appropriate, in general six, degrees of freedom.

In combination with a well-characterized ILMD, such a positioning system can also be used for measuring the partial luminance distribution of a light source. By means of the flexible positioning system, the position of the focus plane of an ILMD with respect to the light source can be varied. In contrast to conventional near-field goniophotometers, therefore a measurement sequence with partly redundant datasets, e.g. by tilting the camera around arbitrary points, can be obtained. Such redundant results are useful for demonstrating critical aspects in sampling of a luminance distribution and related limitations of its reconstruction. The latter are an intrinsic property of the measurement result and of ray data extracted from it. Therefore, these limitations need to be known when using ray data for subsequent simulations, e.g. of a luminaire or illumination scene.

\section{Realized setup}

An established photometric bench is extended by a wall mounted articulated industrial robot with six axes, cf. Figure 3. The drives of these axes are monitored by absolute encoders, giving access to the joint angles. The arm length between shoulder (axis 2) and wrist (axis 5) of the robot is $1,3 \mathrm{~m}$. A safe operation with clearance to the environment (wall, floor, chassis, photometric bench) is ensured via the hardware control by a robust collision model and monitoring of the robot working zone by safety contact mats. In contrast to laser scanners (which represents external sources or light or IR radiation), the used safety system does not disturb the photometric measurement. A more detailed additional check for collisions of the ILMD and robot arm (with each other and with the light source) is used in software-based path planning prior to sending movement commands. 


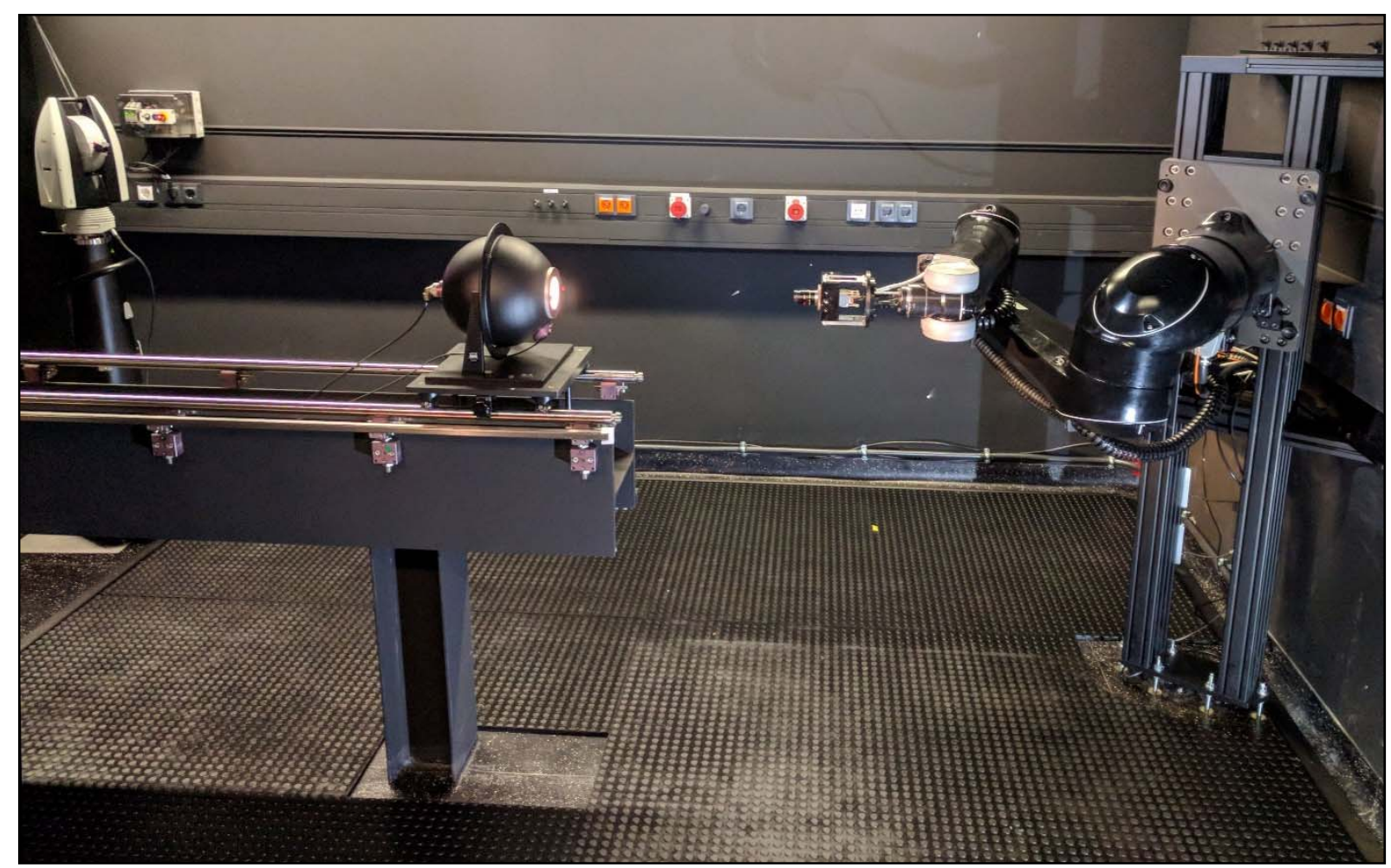

Figure 3 - Photograph of a luminance standard on the photometric bench (centre) observed with an ILMD mounted on the new positioning system (right). The laser tracker (top left) for calibrating the pose stands opposite to the positioning system.

The pose is initially referenced with respect to the existing photometric bench system by using autocollimation and a laser tracker. The wrist flange of the articulated robot is equipped with a stable and repeatable mount for ILMDs with a weight of up to several kilograms. The positioning system provides various data connection standards by means of camera link, GigE and USB to the ILMD.

\section{Aspects of an absolute pose}

Industrial robots are usually employed for the precise repetition of a (relative) pose sequence as programmed by manual teaching. Therefore, such systems are specified with respect to its repeatability. A precise absolute positioning presents an additional challenge. In the installed positioning system this is addressed by an in-field calibration of Denavit-Hartenberg parameters of the kinematic chain and a compensation of gravity related distortions.

The tool centre point (TCP) corresponds to the focus plane of the ILMD which is in a significant distance from the wrist, representing a quite long tool. This gives challenges as small joint angle errors, e.g. due to a backlash of the wrist joints, lead to significant position errors of the TCP. The absolute positioning capabilities are currently under evaluation.

The robot arm segments are in most poses placed behind the ILMD and thus provide a small cross section to the light source, which minimizes interreflections and stray light on the light source. A rotation of the ILMD in up to $1 \mathrm{~m}$ distance to the rotation centre, e.g. corresponding to the light source as in the scheme of Figure 2 right, is possible with a rotation angle with more than $\pm 40^{\circ}$ with respect to the optical axis of the photometric bench.

\section{Conclusions}

The new positioning system extends the photometric bench facility - which is already unique worldwide - by a precise change of the ILMD pose at different measuring distances up to $40 \mathrm{~m}$. This enables a characterization of ILMDs as well as of the partial luminance distribution of a light source and will be advantageous for estimating uncertainty contributions related to their 
spatial and angular characteristic. Although the positioning system is not dedicated for measuring the luminance distribution in a full $2 \pi$ geometry, these insights are beneficial for discussing uncertainties related to conventional near-field goniophotometry.

\section{References}

Bünger, L., Anhalt, K., Taubert, R.D., Krüger, U., Schmidt, F., 2015. Traceability of a CCDCamera System for High-Temperature Measurements. Int. J. Thermophys. 36, 1784-1802. https://doi.org/10.1007/s10765-015-1915-2

Ferrero, A., López, M., Campos, J., Sperling, A., 2014. Spatial characterization of cameras for low-uncertainty radiometric measurements. Metrologia 51, 316-325. https://doi.org/10.1088/0026-1394/51/3/316

Schrader, C., Sperling, A., 2016. Photogrammetrische Verfahren in der bildgebenden Photometrie. Proc. 18. GMA/ITG-Fachtagung Sensoren und Messsyst. 2016 343-350. https://doi.org/10.5162/sensoren2016/5.1.4 